\title{
Erratum: Informing direct neutron capture on tin isotopes near the $N=82$ shell closure [Phys. Rev. C 99, 041302(R) (2019)]
}

B. Manning, G. Arbanas, J. A. Cizewski, R. L. Kozub, S. Ahn, J. M. Allmond, D. W. Bardayan, K. Y. Chae, K. A. Chipps, M. E. Howard, K. L. Jones, J. F. Liang, M. Matos, C. D. Nesaraja, F. M. Nunes, P. D. O’Malley, S. D. Pain, W. A. Peters, S. T. Pittman, A. Ratkiewicz, K. T. Schmitt, D. Shapira, M. S. Smith, and L. Titus

(Received 2 June 2019; published 27 June 2019)

DOI: 10.1103/PhysRevC.99.069901

In Fig. 4 that summarizes calculations of direct-semidirect (DSD) neutron-capture cross sections on tin isotopes at $30 \mathrm{keV}$, we omitted previous work [1] that calculated direct-capture cross sections based on spectroscopic factors reported in Refs. [2-4]. The revised Fig. 4 is provided here. This correction does not affect any of the conclusions presented in the original paper.

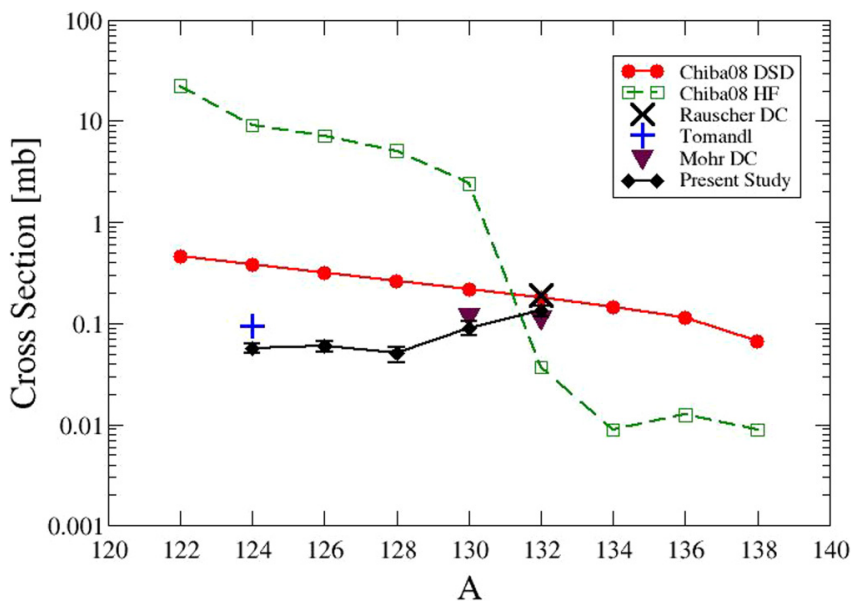

FIG. 4. Comparison of calculated neutron-capture cross sections for tin isotopes at $30 \mathrm{keV}$. Present DSD calculations with experimental energies and spectroscopic factors (black diamonds), Hauser-Feshbach model calculations [5] (green squares), ${ }^{132} \mathrm{Sn}$ direct capture using theoretical spectroscopic factor [6] (black "X"), ${ }^{124}$ Sn DSD calculations using spectroscopic factors of Ref. [7] for all $\ell=1$ levels having $\mathrm{S}>0.01$ (blue "+"), DSD calculations with theoretical excitation energies and spectroscopic factors (red circles) [5], and direct-capture cross sections based on $[3,4]$ spectroscopic factors (purple " $\nabla$ ”) [1].

[1] P. Mohr, Phys. Rev. C 86, 068803 (2012).

[2] K. L. Jones et al., Nature (London) 465, 454 (2010).

[3] K. L. Jones et al., Phys. Rev. C 84, 034601 (2011).

[4] R. L. Kozub et al., Phys. Rev. Lett. 109, 172501 (2012).

[5] S. Chiba, H. Koura, T. Hayakawa, T. Maruyama, T. Kawano, and T. Kajino, Phys. Rev. C 77, 015809 (2008).

[6] T. Rauscher, R. Bieber, H. Oberhummer, K.-L. Kratz, J. Dobaczewski, P. Möller, and M. M. Sharma, Phys. Rev. C 57, 2031 (1998).

[7] I. Tomandl, J. Honzátko, T. von Egidy, H.-F. Wirth, T. Faestermann, V. Yu. Ponomarev, S. Pašić, R. Hertenberger, Y. Eisermann, and G. Graw, Phys. Rev. C 83, 044326 (2011). 